Supporting Information

\title{
A Novel Filler for Gel Polymer Electrolyte with a High Lithium-Ion Transference Number toward Stable Cycling for Lithium-Metal Anodes in Lithium-Sulfur Batteries
}

Huiming Zhang ${ }^{1}$, Huichao Lu ${ }^{1}$, Jiahang Chen ${ }^{1}$, Yanna Nuli ${ }^{1}$, Jiulin Wang ${ }^{* 1,2}$

${ }^{1}$ Department of Chemical Engineering, Shanghai Jiao Tong University, Shanghai 200240, China

${ }^{2}$ College of Chemistry, Zhengzhou University, Henan 450001, China

Email:wangjiulin@sjtu.edu.cn 


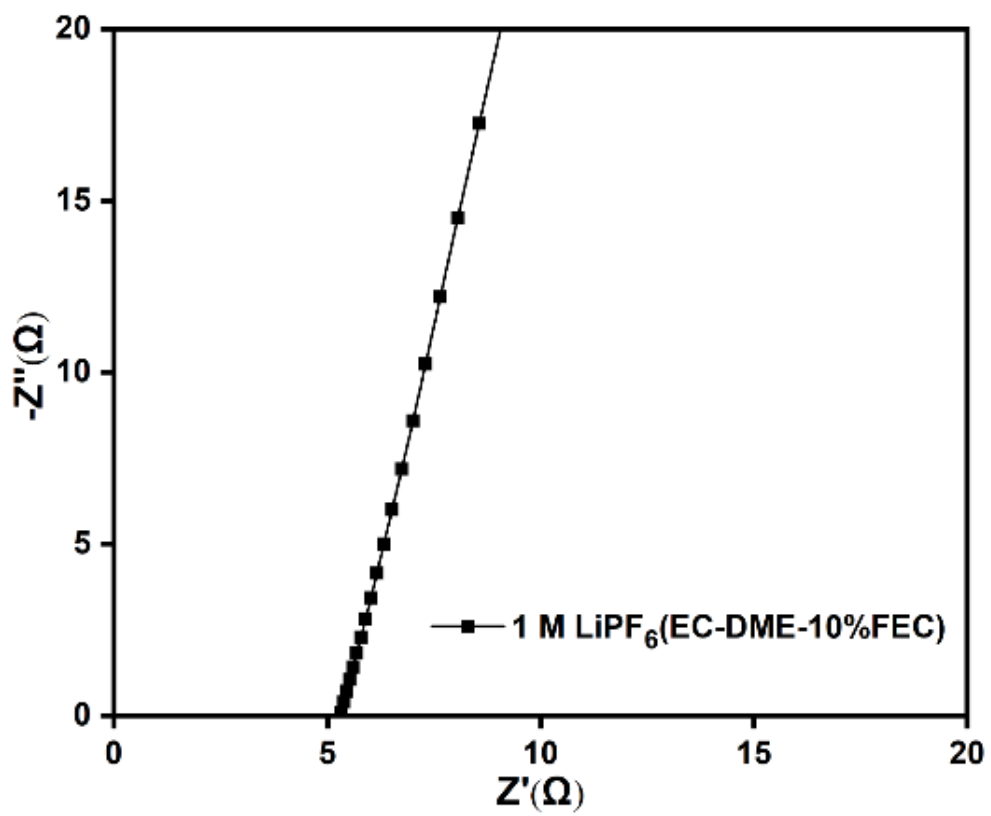

Figure S1. EIS plot of ionic conductivity of SS/PE-liquid electrolyte/SS at ambient temperature. 


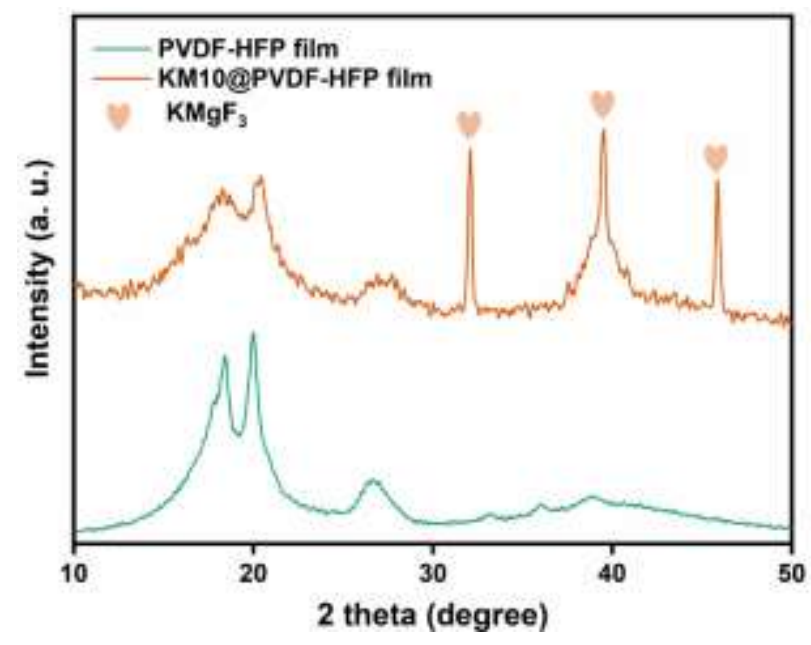

Figure S2. XRD spectra of PVDF-HFP and KM10@PVDF-HFP films.

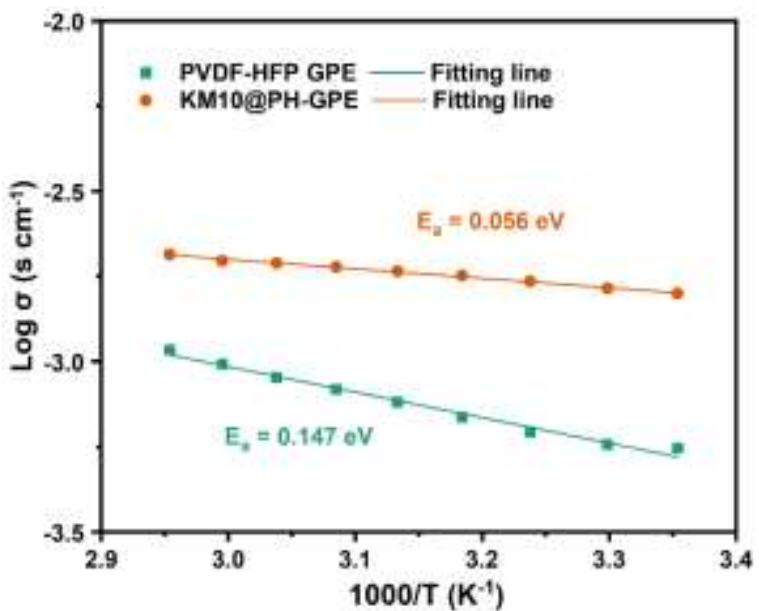

Figure S3. Temperature dependent ion conductivity of PVDF-HFP GPE and KM10@PH-GPE from 25 to $65^{\circ} \mathrm{C}$. 

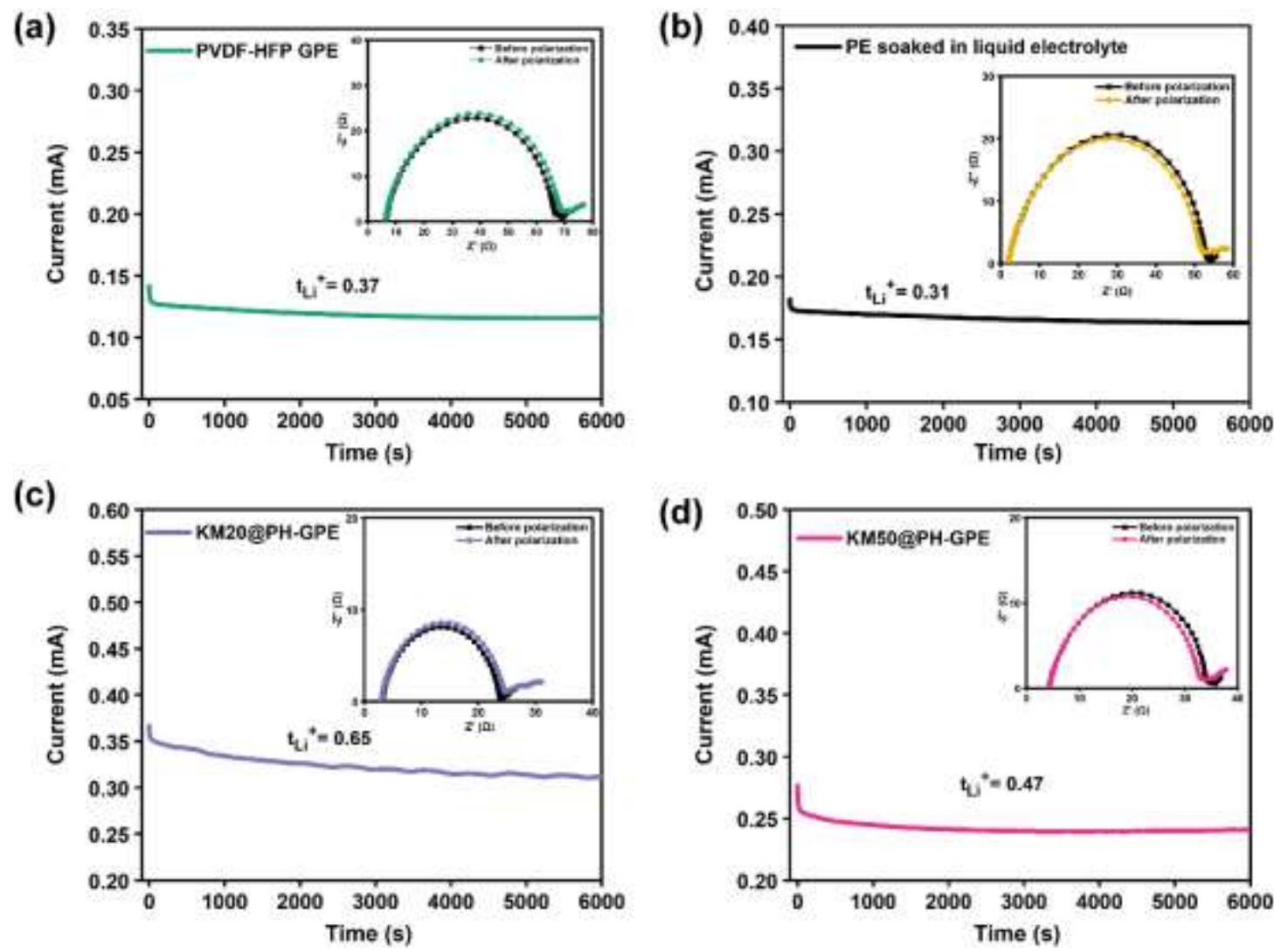

Figure S4. Chronoamperometry curves of (a) Li/ PVDF-HFP GPE/Li, (b) Li/ PE soaked in liquid electrolyte /Li, (c) Li/ KM20@PH-GPE /Li and (d) Li/ KM50@PH-GPE /Li with a static potential of $10 \mathrm{mV}$. The corresponding EIS plots before and after polarization are inserted.

(a)

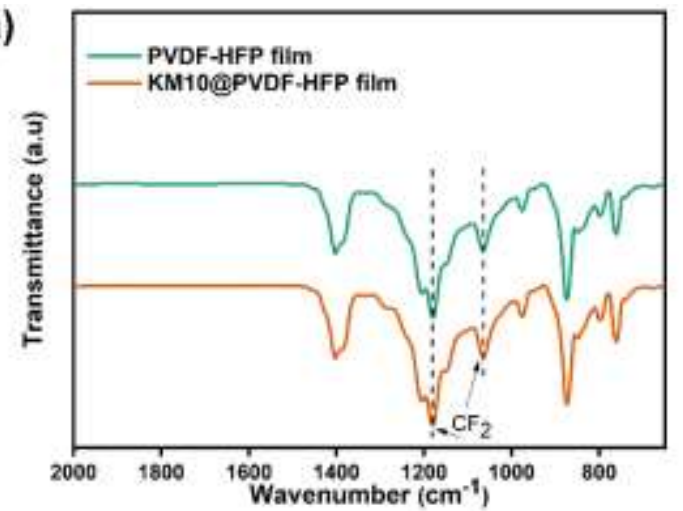

(b)

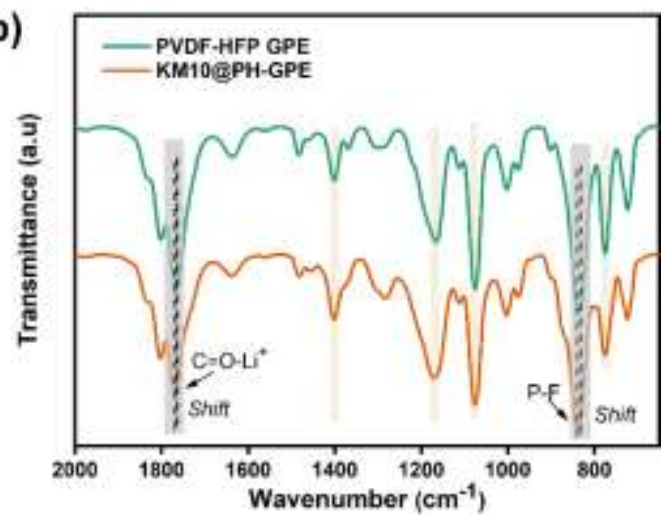

Figure S5. (a) FTIR spectra of PVDF-HFP and KM10@PVDF-HFP films. (b) FTIR 
spectra of PVDF-HFP GPE and KM10@PH-GPE. The peaks from PVDF-HFP are marked with a light yellow background.

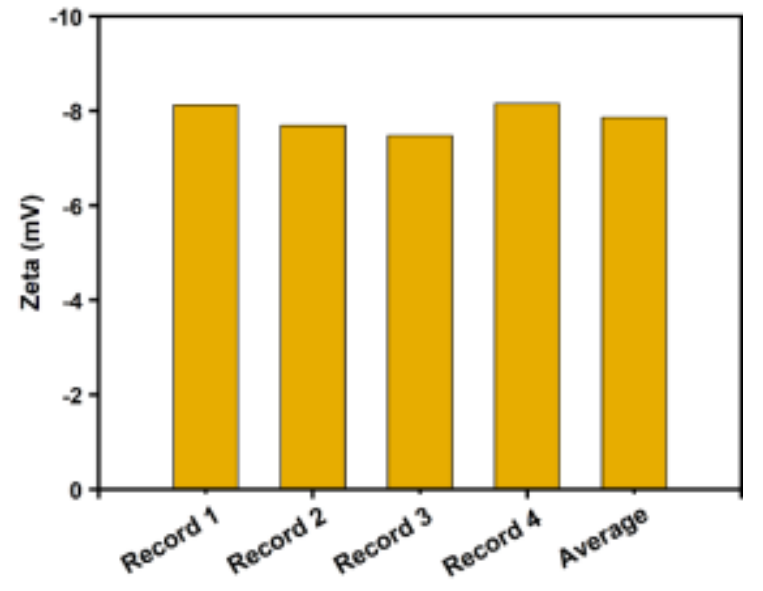

Figure S6. Zeta potential of $\mathrm{KMgF}_{3}$ in carbonate solvent.
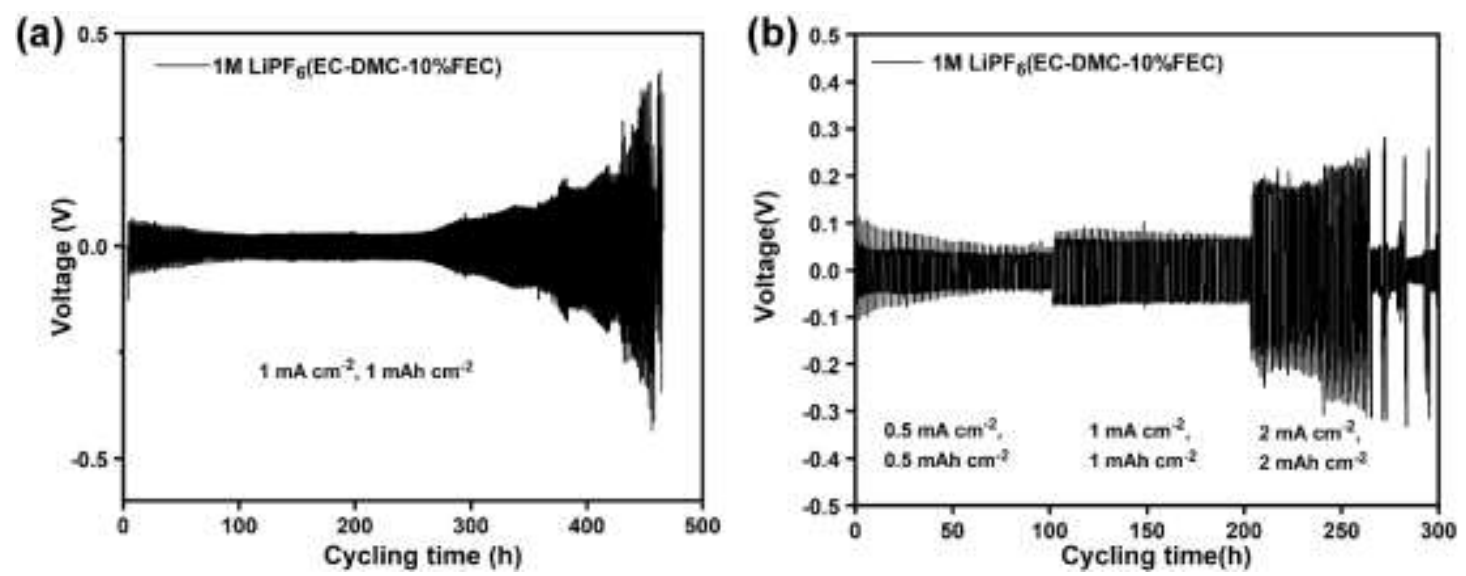

Figure S7. (a) The cycling performance of Li symmetric battery with PE soaked in liquid electrolyte at the current density of $1 \mathrm{~mA} \mathrm{~cm}^{-2}$ with a capacity of $1 \mathrm{~mA} \mathrm{~h} \mathrm{~cm}$. (b) Rate performances of $\mathrm{Li}$ symmetric battery with liquid electrolyte at various current densities from 0.5 to $2 \mathrm{~mA} \mathrm{~cm}^{-2}$. 

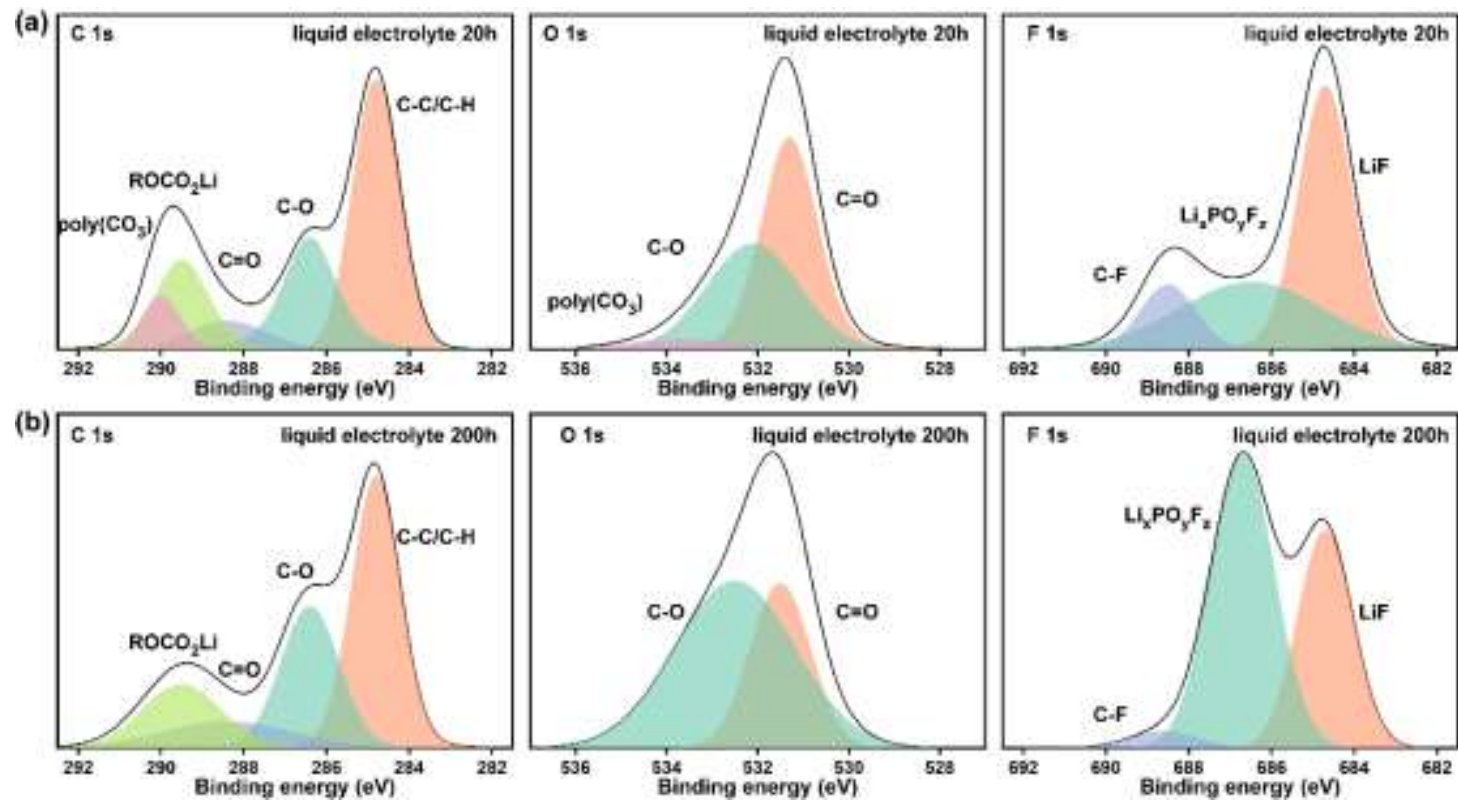

Figure S8. XPS spectrums of Li metal after (a) 20 hours and (b) 200 hours using liquid electrolyte.
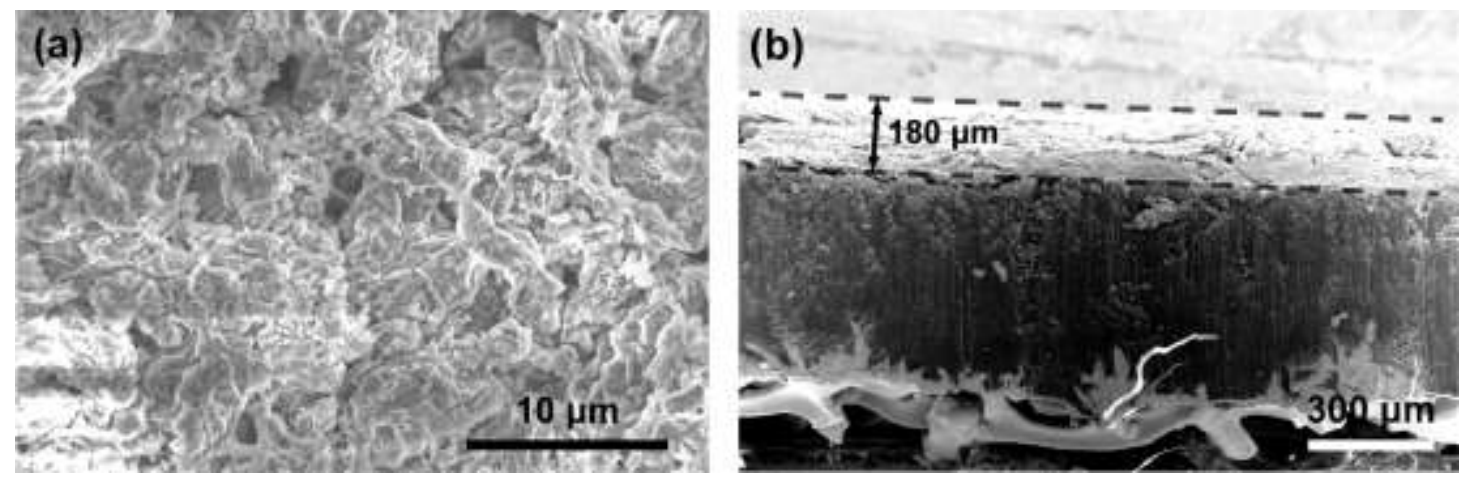

Figure S9. SEM images of lithium metal anode from Li symmetric batteries using liquid electrolyte after cycling for $200 \mathrm{~h}$ at $1 \mathrm{~mA} \mathrm{~cm}^{-2}$ from (a) surface and (b) 
cross-sectional views.

Table S1. Thickness of KMx@PH and PVDF-HFP films

\begin{tabular}{|c|c|c|c|c|}
\hline film & PVDF-HFP & KM10@PH & KM20@PH & KM50@PH \\
\hline Thickness $(\mu \mathrm{m})$ & 20 & 40 & 45 & 52 \\
\hline
\end{tabular}

Table S2. Comparison of ion conductivity and lithium ion transference number of different polymer electrolytes

\begin{tabular}{|c|c|c|c|c|}
\hline References & Electrolytes & Temperature & $\sigma\left(\mathrm{mS} \mathrm{cm}^{-1}\right)$ & $\mathbf{t}_{\mathrm{Li}}^{+}$ \\
\hline 1 & $\begin{array}{c}\text { PVdF-HFP/PYRA }{ }_{120} \\
{ }_{1} \text { TFSI/LiTFSI/HiSil }^{\text {T }} \\
{ }_{\text {M }}{ }_{\text {T700 }}\end{array}$ & $20{ }^{\circ} \mathrm{C}$ & 0.333 & 0.21 \\
\hline 2 & PVDF/HEC/PVDF & $25^{\circ} \mathrm{C}$ & 0.88 & 0.57 \\
\hline 3 & $\begin{array}{c}\text { PDMA-silica/Li(G4) } \\
\text { TFSI }\end{array}$ & $25^{\circ} \mathrm{C}$ & 0.12 & 0.43 \\
\hline
\end{tabular}




\begin{tabular}{|c|c|c|c|c|}
\hline 4 & $\begin{array}{c}\text { LiTFSI/EMIM-TFSI } \\
\text { /hBN }\end{array}$ & $25^{\circ} \mathrm{C}$ & $>1$ & 0.18 \\
\hline 5 & $\begin{array}{c}\text { PIL-UPy/DE-IM/TF } \\
\text { SI ILs /LiTFSI }\end{array}$ & $25^{\circ} \mathrm{C}$ & 1.41 & 0.26 \\
\hline 6 & $\begin{array}{c}\text { Methyl } \\
\text { propenyl-fumed } \\
\text { SiO } / \text { /VC/ETPTA }\end{array}$ & $25^{\circ} \mathrm{C}$ & 0.41 & 0.54 \\
\hline 7 & $\begin{array}{c}\text { POSS/PEG/Pyr13FS } \\
\text { I/LiFSI }\end{array}$ & $20^{\circ} \mathrm{C}$ & 1.22 & 0.48 \\
\hline 8 & $\begin{array}{c}\text { PVDF-HFP/NMP/Li } \\
\text { TFSI }\end{array}$ & $25^{\circ} \mathrm{C}$ & 0.121 & 0.57 \\
\hline 9 & PCUMA-GPE & $30{ }^{\circ} \mathrm{C}$ & 1.27 & 0.44 \\
\hline This work & KM10@PH-GPE & $25{ }^{\circ} \mathrm{C}$ & 1.63 & 0.63 \\
\hline
\end{tabular}

\section{References}

(1) Ferrari, S.; Quartarone, E.; Mustarelli, P.; Magistris, A.; Fagnoni, M.; Protti, S.; Gerbaldi, C.; Spinella, A. Lithium Ion Conducting PVdF-HFP Composite Gel Electrolytes Based on N-methoxyethyl-N-Methylpyrrolidinium Bis(trifluoromethanesulfonyl)-Imide Ionic Liquid. J. Power Sources 2010, 195, 559-566, DOI: 10.1016/j.jpowsour.2009.08.015.

(2) Zhang, M. Y.; Li, M. X.; Chang, Z.; Wang, Y. F.; Gao, J.; Zhu, Y. S.; Wu, Y. P.; Huang, W. A Sandwich PVDF/HEC/PVDF Gel Polymer Electrolyte for Lithium Ion Battery. Electrochim. Acta 2017, 245, 752-759, DOI: 10.1016/j.electacta.2017.05.154.

(3) Yu, L.; Guo, S.; Lu, Y.; Li, Y.; Lan, X.; Wu, D.; Li, R.; Wu, S.; Hu, X. Highly Tough, Li-Metal Compatible Organic-Inorganic Double-Network Solvate Ionogel. Adv. Energy Mater. 2019, 9, 1900257, DOI: 10.1002/aenm.201900257.

(4) Hyun, W. J.; de Moraes, A. C. M.; Lim, J. M.; Downing, J. R.; Park, K. Y.; Tan, M. T. Z.; Hersam, M. C. High-Modulus Hexagonal Boron Nitride Nanoplatelet Gel Electrolytes for Solid-State Rechargeable Lithium-Ion Batteries. ACS Nano 2019, 13, 
9664-9672, DOI: 10.1021/acsnano.9b04989.

(5) Guo, P.; Su, A.; Wei, Y.; Liu, X.; Li, Y.; Guo, F.; Li, J.; Hu, Z.; Sun, J. Healable, Highly Conductive, Flexible, and Nonflammable Supramolecular Ionogel Electrolytes for Lithium-Ion Batteries. ACS Appl. Mater. Interfaces 2019, 11, 19413-19420, DOI: 10.1021/acsami.9b02182.

(6) Li, H.; Yang, J.; Xu, Z.; Lu, H.; Zhang, T.; Chen, S.; Wang, J.; NuLi, Y.; Hirano, S.-i. Integrated Composite Polymer Electrolyte Cross-Linked with $\mathrm{SiO}_{2}$-Reinforced Layer for Enhanced Li-Ion Conductivity and Lithium Dendrite Inhibition. ACS Appl. Energy Mater. 2020, 3, 8552-8561, DOI: 10.1021/acsaem.0c01173.

(7) Li, X.; Zheng, Y.; Li, C. Y. Dendrite-Free, Wide Temperature Range Lithium Metal Batteries Enabled by Hybrid Network Ionic Liquids. Energy Storage Mater. 2020, 29, 273-280, DOI: 10.1016/j.ensm.2020.04.037.

(8) Jie, J.; Liu, Y.; Cong, L.; Zhang, B.; Lu, W.; Zhang, X.; Liu, J.; Xie, H.; Sun, L. High-performance PVDF-HFP based gel polymer electrolyte with a safe solvent in Li metal polymer battery. J. Energy Chem. 2020, 49, 80-88, DOI: 10.1016/j.jechem.2020.01.019.

(9) Hu, R.; Qiu, H.; Zhang, H.; Wang, P.; Du, X.; Ma, J.; Wu, T.; Lu, C.; Zhou, X.; Cui, G. A Polymer-Reinforced SEI Layer Induced by a Cyclic Carbonate-Based Polymer Electrolyte Boosting $4.45 \mathrm{~V} \mathrm{LiCoO}_{2} / \mathrm{Li}$ Metal Batteries. Small 2020, 16, e1907163, DOI: 10.1002/smll.201907163.

Table S3. Elemental analysis of S@pPAN composite materials

\begin{tabular}{|c|c|c|c|c|}
\hline Element & N \% & C \% & H \% & S \% \\
\hline S@pPAN & 13.97 & 38.05 & 0.98 & 44.57 \\
\hline
\end{tabular}

